\title{
Allowable Forward Model Misspecification for Accurate Basis Decomposition in a Silicon Detector Based Spectral CT
}

\author{
Hans Bornefalk*, Mats Persson, and Mats Danielsson
}

\begin{abstract}
Material basis decomposition in the sinogram domain requires accurate knowledge of the forward model in spectral computed tomography (CT). Misspecifications over a certain limit will result in biased estimates and make quantum limited (where statistical noise dominates) quantitative $\mathrm{CT}$ difficult. We present a method whereby users can determine the degree of allowed misspecification error in a spectral CT forward model and still have quantification errors that are limited by the inherent statistical uncertainty. For a particular silicon detector based spectral CT system, we conclude that threshold determination is the most critical factor and that the bin edges need to be known to within $0.15 \mathrm{keV}$ in order to be able to perform quantum limited material basis decomposition. The method as such is general to all multibin systems.
\end{abstract}

Index Terms-Accuracy, forward model, photon counting multibin computed tomography (CT), quantification, spectral CT.

\section{INTRODUCTION}

$\mathbf{S}$ PECTRAL computed tomography (CT) systems allows for, among other things, quantitative CT using basis decompositions methods [1], [2]

$$
\mu(\mathbf{r} ; E)=a_{1}(\mathbf{r}) f_{1}(E)+a_{2}(\mathbf{r}) f_{2}(E) .
$$

Equation (1) indicates that the entire energy dependence of the linear attenuation for each voxel can be determined and not just an energy weighted average. There are several ways in which such a decomposition can be carried out. If only two bases are used in (1) dual energy systems, with two independent measurements, give adequate energy resolving power to allow for basis decomposition. If more bases are desired, or higher precision of the basis coefficients needed, energy sensitive multibin systems can be applied. The decomposition could be performed in image space but this would not eliminate beam hardening artifacts. Therefore a projection based approach is desired [3].

There are two basic methods whereby such a decomposition in the projection domain can be carried out. One is by means of

\footnotetext{
Manuscript received August 22, 2014; accepted September 28, 2014. Date of publication October 09, 2014; date of current version February 27, 2015. This work was supported by the Erling-Persson Family Foundation. Asterisk indicates corresponding author.

*H. Bornefalk is with the Department of Physics, Royal Institute of Technology, AlbaNova University Center, SE-106 91 Stockholm, Sweden (e-mail: hans.bornefalk@mi.physics.kth.se).

M. Persson and M. Danielsson are with the Department of Physics, Royal Institute of Technology, AlbaNova University Center, SE-106 91 Stockholm, Sweden.
}

Digital Object Identifier 10.1109/TMI.2014.2361680 calibration measurements where known combinations of calibration material thicknesses is used to obtain a set of detector responses against which actual measurements can be "compared" (polynomially fitted [4] or otherways interpolated [5]). A second approach is to directly determine the line integrals of $a_{i}(\mathbf{r})$ from the sinogram data using a maximum likelihood approach and then reconstruct $a_{i}$ 's using the inverse Radon transform. Such an approach for multibin systems has been presented by Röß1 and Herrmann [7] and successfully applied to real data on a prototype system by Rößl and Proksa [8].

This approach requires accurate knowledge of the forward model, otherwise bias is introduced in the estimates [3], [6]. The forward model is the physical model describing the signal generation in the detector. For a photon counting multibin system, the expected number of counts in bin $i$, i.e., events with deposited energy $E^{\prime}$ in the range $\left[T_{i-1}, T_{i}\right)$ is given by

$$
\begin{aligned}
\lambda_{i}\left(x^{\prime}, \theta\right)=N_{0} \int_{0}^{\infty} \Phi(E) & D(E) S_{i}(E) \\
& \times \mathrm{e}^{-\int_{l} \mu(\mathbf{r} ; E) \mathrm{d} s} \mathrm{~d} E+\varepsilon_{i}\left(x^{\prime}, \theta\right)
\end{aligned}
$$

where

$$
S_{i}(E)=\int_{T_{i-1}}^{T_{i}} R\left(E, E^{\prime}\right) \mathrm{d} E^{\prime}
$$

and $\varepsilon_{i}\left(x^{\prime}, \theta\right)$ is the expected number of scatter counts in bin $i$. $N_{0}$ is the expected value of the number of photons, in the unattenuated beam, hitting a detector element $x^{\prime}$ at a rotation angle $\theta$. The path $l$ connects the X-ray source with the detector element $x^{\prime}, \Phi(E)$ denotes the energy distribution of the X-ray spectrum, $D(E)$ the detection efficiency. Following the notation of Röß1 and Herrmann [7] $R\left(E, E^{\prime}\right)$ denotes the probability that an X-ray photon with energy $E$ deposits charge corresponding to an energy $E^{\prime}$ in the detector and $S_{i}(E)$ is therefore a bin function, giving the fraction of photons with energy $E$ that are deposited in the $i$ th bin. The parameters of the forward model are thus $N_{0}, \Phi(E), D(E), R\left(E, E^{\prime}\right), \varepsilon_{i}\left(x^{\prime}, \theta\right)$ and $T_{i}, i=1, \ldots, N$, where $N$ is the number of energy bins. $\left(N_{0}\right.$, the unattenuated fluence, is assumed to be measurable with high precision.)

This investigation is concerned with to which accuracy the parameters of the forward model must be known in order to perform material basis decomposition in practice. The approach differs from that taken by Walter et al. [6] in at least two aspects. The first is that not only the spectral shape $\Phi$ is consid- 
ered, but also other parameters, especially those that are specific to multibin systems like the uncertainty in the set of internal threshold $\left\{T_{i}\right\}$ and the energy response function (the previous investigations were concerned with dual energy methods). The second is the figure of merit applied. When the parameters of the forward model are incorrect the estimates $\hat{a}_{1}(\mathbf{r})$ and $\hat{a}_{2}(\mathbf{r})$ will be biased. This bias will add to the variance and result in a mean square error that is larger than the variance of the unbiased estimate $\left(E[\cdot]\right.$ is the expectation operator and the hat, ${ }^{\wedge}$, denotes an estimate)

$$
\begin{aligned}
\operatorname{MSE}(\hat{a}) & =E\left[(\hat{a}-a)^{2}\right]=E\left[\hat{a}^{2}\right]-2 E[\hat{a}] a+a^{2} \\
& =\left(E\left[\hat{a}^{2}\right]-E[\hat{a}]^{2}\right)+(E[\hat{a}]-a)^{2} \\
& =\operatorname{var}(\hat{a})+\operatorname{bias}^{2}(\hat{a}) .
\end{aligned}
$$

We determine $\operatorname{MSE}(\hat{a})$ for different deviations of the forward parameters from the true values and relate them to the inherent uncertainty, the Cramér-Rao lower bound of the variance, that would remain had the forward model not been misspecified. As a cutoff, we determine the allowable misspecification for each parameter of the forward model would result in a squared bias equal to the variance.

The method is developed and illustrated for a photon counting multibin system with silicon detector diodes [9]-[12] but the methodology applies equally well to other detector materials. A shorter version of this paper has been presented in a conference proceeding [13].

\section{BACKGROUND}

Since the detection efficiency, response function and spectral shape are continuous functions (or vectorized quantities in practice), assigning a small perturbation or misspecification error to them is nontrivial and can be done in a multitude of ways. Just like Walter et al. [6] we derive reasonable misspecification errors of the forward model by 'first principles'. For example, just like they do, we let a change in X-ray tube acceleration voltage and filter thickness affect the spectral shape. This section is devoted to illustrating how we arrive at misspecifications for the various forward model parameters.

A comment on an underlying model assumption might be in place here: we do not model the parameters of the forward equation as variable over time. They are assumed fixed but unknown. It is how the error in their determination should be modeled and how large the accompanying uncertainty can be before the material basis decomposition breaks down that we want to determine.

\section{A. Modeling the Uncertainty in Scatter Counts $\varepsilon_{i}\left(x^{\prime}, \theta\right)$}

To perform accurate basis decomposition according to (3), the scatter in each projection must be estimated. As scatter is a spatially slowly varying function, it can be estimated by shielding a fraction of the detector elements from primary rays and thus have them only estimate scatter. Such an approach will inevitably result in some misspecification of the scatter, and we are interested in determining how accurate the estimation must be. In this work we assume the scatter-to-primary ratio of detected counts is $\mathrm{SPR}_{0}=0.15$ in the forward model, and then examine the resulting bias when the actual ratio SPR differs. $15 \%$ scatter is a tentative estimate for the modeled silicon

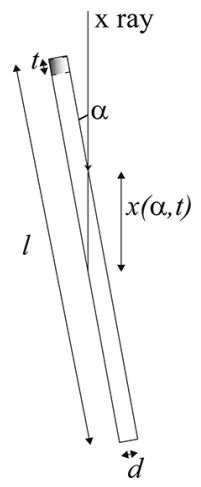

Fig. 1. Effective absorbtion length $x$ as a function of misalignment angle $\alpha$ and dead layer thickness $t$.

detector system; 8\% internal detector scatter [9] and a similar amount of object scatter. This allows us to draw reasonable conclusions about how much the model assumptions can be allowed to deviate from the truth. (Please note that while the implementation, to mimic reality, assumes a certain number of scatter counts in each energy bin, this number is expressed as a ratio to the signal count for ease of interpretation.)

\section{B. Modeling the Uncertainty of the Detection Efficiency $D(E)$}

To increase the detection efficiency of the silicon diodes, they $\mathrm{X}$-rays enter edge on, i.e., in the plane of the wafer they are cut from [9]. This requires a careful alignment. Any misalignment, as captured by the angle $\alpha$ results in a shorter attenuating distance $x$ (cf. Fig. 1) in the silicon. There is also an uncertainty regarding the exact thickness $t$ of the dead layer depicted in the figure. Somewhat simplified, $x(\alpha, t) \approx \min (l-t, d / \sin \alpha)$. One could argue that this underestimates the active length for misalignment errors larger than $\alpha^{*}=\sin ^{-1}(d /(l-t))$, the misalignment angle for which $l-t=d / \sin \alpha$, since transmitted $\mathrm{X}$-rays could interact in adjacent, presumably also misaligned, strips. However, for $d=0.5 \mathrm{~mm}, l=32 \mathrm{~mm}$, and $t \approx 0.5 \mathrm{~mm}$, $\alpha^{*} \approx 0.9^{\circ}$ and this is a rather large angle and any high accuracy alignment procedure can be expected to result in errors smaller than this. For that reason, we model the uncertainty in the detection efficiency as only being dependent on the thickness $t$ of the dead layer

$$
D(E ; t)=\mathrm{e}^{-\mu(E) t}\left(1-\mathrm{e}^{-\mu(E)(l-t)}\right)
$$

where $\mu(E)$ is the linear attenuation coefficient of silicon (only the attenuation from incoherent scattering and photoelectric effect is included). $t=0.5 \mathrm{~mm}$ and $\Delta t$, the modeled deviation from the assumed value of $t$, ranging from -0.2 to $+0.2 \mathrm{~mm}$, yield the detection efficiency curves in the left panel of Fig. 2.

The error in the assumed linear attenuation coefficient $\mu(E)$ for silicon is estimated to be $1 \%$ or less in the literature [14], [15]. This relative uncertainty is of the same order of magnitude as $\Delta t /(l-t)$ and as both parameters are multiplied in the exponent of (5), the uncertainty in the linear attenuation coefficient ought also to be considered. In the right panel of Fig. 2, the detection efficiency for $\Delta t=0 \mathrm{~mm}$ is shown for a $1 \%$ overall increase and $1 \%$ overall decrease in $\mu(E)$ compared to tabulated values in XCOM [15]. 

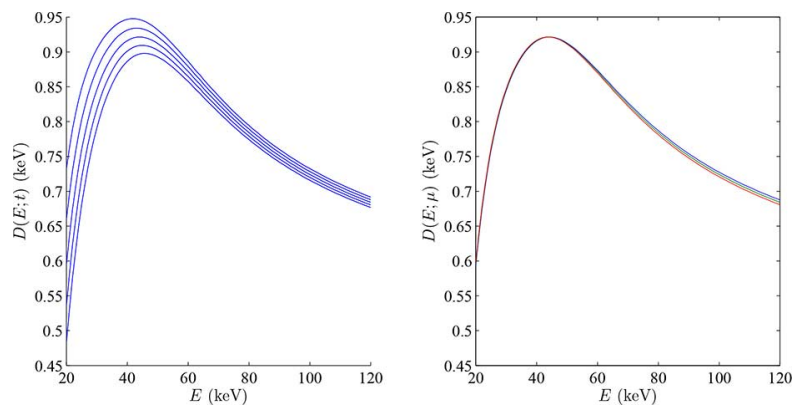

Fig. 2. Left panel: Detection efficiencies according to (5) for, from top to bottom, $t=0.3,0.4,0.5,0.6$ and $0.7 \mathrm{~mm}$. Right panel: $D(E)$ for $t$ fixed at $0.5 \mathrm{~mm}$ and, from top to bottom, $\mu(E)=1.01 \mu_{0}(E), \mu_{0}(E)$ and $0.99 \mu_{0}(E)$ where $\mu_{0}(E)$ is the tabulated XCOM value.

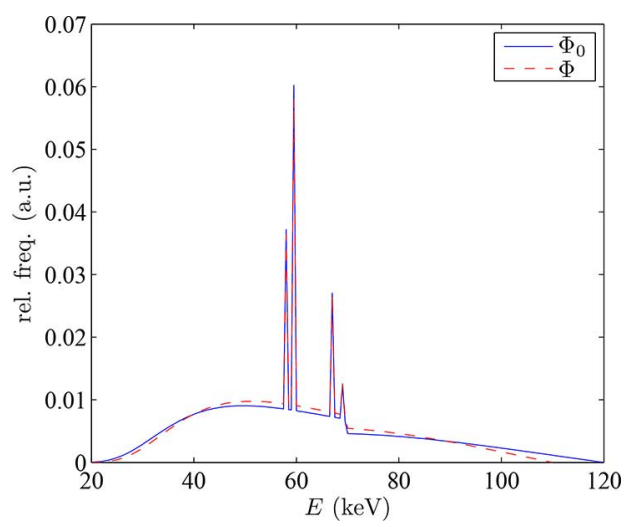

Fig. 3. Illustration of exaggerated spectral distortion resulting from lower acceleration voltage and increased aluminum filtration.

\section{Modeling the Uncertainty of the X-Ray Spectrum $\Phi(E)$}

We use tungsten X-ray spectra as modeled by Cranley et al. [16]. For the default spectrum $\Phi_{0}(E) U_{0}=120 \mathrm{kV}$ acceleration voltage and $d_{0, A l}=6 \mathrm{~mm}$ aluminum filtration is assumed.

When the actual acceleration voltage is $U$ and the aluminum thickness $d_{A l}$, the $\mathrm{X}$-ray spectrum $\Phi\left(E ; U, d_{A l}\right)$ will be different from $\Phi_{0}(E)$ and this is how we model uncertainties in the spectral shape.

In Fig. 3, $\Phi\left(E ; U, d_{A l}\right)$ and $\Phi_{0}(E)$ are depicted for $U=110$ $\mathrm{kV}$ and $d_{A l}=4 \mathrm{~mm}$ (greatly exaggerated deviations from model for illustrative purposes).

\section{Modeling the Uncertainty of the Reference Thresholds $\left\{T_{i}\right\}$}

As an interacting photon deposits energy (in units of keV) in the diode material, charge carries are generated which are subsequently fed through an analog circuit to generate a voltage pulse (in units of $\mathrm{mV}$ ). The relationship between energy and pulse height is linear

$$
u_{j}(E)=g_{j} E+m_{j}
$$

where index $j$ indicates that the response is channel dependent, i.e., differs between channels.

When the set of thresholds $\left\{T_{i}\right\}, i=1, \ldots, N$ are implemented, $N$ global (i.e., channel independent) reference voltages $U_{i}$ are applied to the $N$ discriminators [17]. Due to the channel variation of the gain $g_{j}$ (in units $\mathrm{mV} / \mathrm{keV}$ ) and the offset $m_{j}$ (in units $\mathrm{mV}$ ), these reference voltages will correspond to different deposited energies in each channel (which can be thought of as a detector element)

$$
T_{i j}=\left(U_{i}-m_{j}\right) / g_{j} .
$$

In an energy weighted image, such channel inhomogeneities would result in ring artifacts if not properly compensated for [18] and [19]. When material basis decomposition is applied to the sinogram data, the inhomogeneities require that different forward equation parameters are used for each channel $j$, according to (7). A problem of misspecification might occur however since $m_{j}$ and $g_{j}$ are only known up to certain precision (and their values are known to drift with temperature, albeit in a predictable fashion) [10].

In the ideal case, the gain $g_{j}$ (in units $\mathrm{mV} / \mathrm{keV}$ ) and offset $m_{j}$ (in units $\mathrm{mV}$ ) for each channel can be determined from a synchrotron source, as done in previous work [11], [12], [10]. In the limit case of infinite exposure the statistical error of the estimates will approach zero. Under one set of typical synchrotron radiation exposure conditions [10], the relative errors $\Delta g$ and $\Delta m$ (i.e., the estimated uncertainty in the estimates of $\hat{g}_{j}$ and $\hat{m}_{j}$ due to finite statistics) was $\Delta g=0.019 \mathrm{mV} / \mathrm{keV}$ and $\Delta m=$ $0.55 \mathrm{mV}$. This should be compared to the average gain and offset vales of $\overline{\hat{g}}=1.64 \mathrm{mV} / \mathrm{keV}$ and $\overline{\hat{m}}=6.87 \mathrm{mV}$ [10]. The uncertainties $\Delta g$ and $\Delta m$ translate to an uncertainty $\Delta T_{i}$ of the bin thresholds. For a threshold at $T_{i}=30 \mathrm{keV}$ this uncertainty is approximated by (not taking correlation between $g$ and $m$-estimates into consideration which would lower the estimate)

$$
\begin{aligned}
\left|\Delta T_{i}\right| & =T_{i} \sqrt{\left(\frac{\Delta m}{U_{i}-\overline{\hat{m}}}\right)^{2}+\left(\frac{\Delta g}{\overline{\hat{g}}}\right)^{2}} \\
& =T_{i} \sqrt{\left(\frac{\Delta m}{T_{i} \overline{\hat{g}}}\right)^{2}+\left(\frac{\Delta g}{\overline{\hat{g}}}\right)^{2}}
\end{aligned}
$$

which evaluates to $0.48 \mathrm{keV}$. We model the threshold uncertainty effect by evaluating the bias for uncertainties in the thresholds ranging from $\Delta T=-1 \mathrm{keV}$ to $1 \mathrm{keV}$. All thresholds are assumed to move in parallel by $\Delta T$, which is a likely consequence of a global change in temperature [10].

\section{E. Modeling the Uncertainty of the Response Function $R\left(E, E^{\prime}\right)$}

The derivation of the response function for a silicon strip detector system has been presented in the literature [9]. Given that an interaction of a photon of energy $E$ has occurred, the fraction that undergoes photoelectric interaction to the fraction of Compton interactions is given by the ratio of the corresponding photon cross sections. From the above section we now expect that there is an uncertainty in tabulated value of these cross sections.

For Compton interactions the distribution of deflection angles is given by the Klein-Nishina differential cross section, and this approach assumes free outer shell electrons. This is a second potential source of error. Given the X-ray photon deflection angle and an initial electron at rest, the deposited energy is obtained via the Compton scatter formula.

Since charge generation is a stochastic process, the generated charge and thus the measurable energy deposition, follows a distribution around the value predicted by the photoelectric effect 
and the Compton scatter formula. The deviation from Poisson statistics (the actual distribution is narrower) is captured by the Fano factor and its accurate determination is elusive [20]. Thus different tabulated values exist for silicon, in the vicinity of $F$ $=0.11$. This introduces an additional source of possible forward model specification error.

All generated charge carriers are not collected in the correct detector element as some dissipate to neighboring detector elements. The extent of this charge sharing is highly stochastic and depends on interaction location and initial charge cloud size and shape. For events depositing charge at the border of a neighboring pixel the fraction of the charge collected in the correct pixel have an expected value of $50 \%$ and this results in a highly leftward-skewed distribution of detected energies around the deposited energy. This distribution can be estimated [9] by making assumptions regarding electric field strength, charge drift velocity and diffusion speed but such modeling is prone to errors (for instance the diffusion coefficient depends on temperature which cannot be assumed to be known exactly). To complicate matters more, pile-up at high photon flux will also distort the collected energy spectrum [21], by a degree varying with the flux. The channel dependent electronic noise, which can only be ascertained with a certain precision, of course also presents an obstacle to the exact determination of the response function.

Recall that the purpose of this investigation is to determine which degree of model misspecification is acceptable before the material basis decomposition method breaks down. For this purpose, it is actually not important to model the correct response function in detail - it is the deviation from the assumed that matters and this deviation must be quantifiable.

Let $\gamma_{0}\left(E, E^{\prime \prime}\right)$ be the true, but unknown, distribution of initially released energy $E^{\prime \prime}$ from the primary interaction of a photon with energy $E$. Now let $h_{0}\left(E^{\prime}, E^{\prime \prime}\right)$ be a distribution that captures charge sharing, pile-up, the Fano-spreading and the electronic noise and, for an initial deposition of $E^{\prime \prime}$ in a detector element, yields the probability of collecting $E^{\prime}$. The real response function is now

$$
R_{0}\left(E, E^{\prime}\right)=\int_{-\infty}^{\infty} \gamma_{0}\left(E, E^{\prime \prime}\right) h_{0}\left(E^{\prime}, E^{\prime \prime}\right) \mathrm{d} E^{\prime \prime} .
$$

It holds that $\int \gamma_{0}\left(E, E^{\prime \prime}\right) \mathrm{d} E^{\prime \prime}=\int h_{0}\left(E^{\prime}, E^{\prime \prime}\right) \mathrm{d} E^{\prime}=$ $\int R_{0}\left(E, E^{\prime}\right) \mathrm{d} E^{\prime}=1$.

When we want to estimate the response function by $R\left(E, E^{\prime}\right)$, a first step is to determine $\gamma\left(E, E^{\prime \prime}\right)$ by taking the probabilities of photoelectric effect and Compton scatter into consideration and, for Compton events, the Compton scatter formula [9]. A next step would be to estimate the degradation due to pile-up, charge sharing, the statistics of electron-hole formulation and electronic noise as captured by $h\left(E^{\prime}, E^{\prime \prime}\right)$, our estimate of $h_{0}$, and form $R\left(E, E^{\prime}\right)=\int_{-\infty}^{\infty} \gamma\left(E, E^{\prime \prime}\right) h\left(E^{\prime}, E^{\prime \prime}\right) \mathrm{d} E^{\prime \prime}$. It should hold that $\int R\left(E, E^{\prime}\right) \mathrm{d} E^{\prime}=1$.

The distribution of deflection angles $\theta$ are given by (10) [9]

$$
\begin{aligned}
& \frac{d \sigma}{d \theta} \propto\left(1+\cos ^{2} \theta+\frac{k^{2}(1-\cos \theta)^{2}}{1+}\right. k(1-\cos \theta) \\
& \times(1+k(1-\cos \theta))^{-2} \sin \theta
\end{aligned}
$$
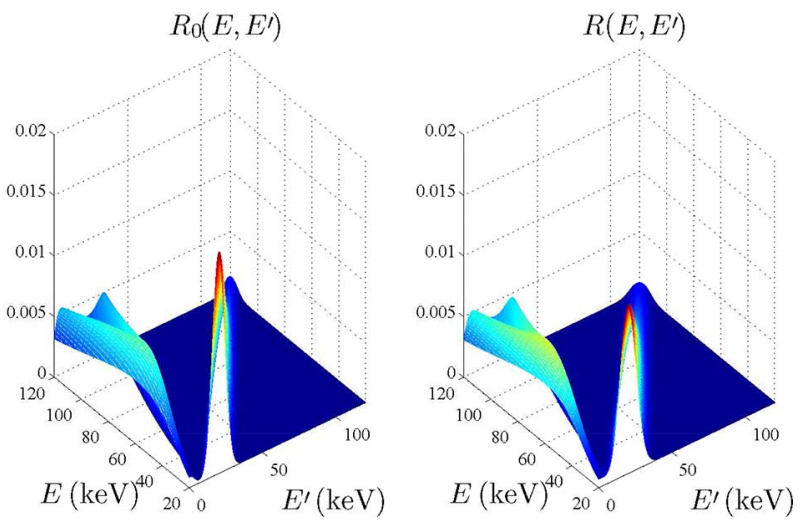

Fig. 4. Response functions. $R$ is the faulty approximation of $R_{0}$ obtained by assuming a internal energy resolution from charge sharing, pile-up and electronic noise ( $h$ being a Gaussian with $4 \mathrm{keV}$ standard deviation instead of $h_{0}$, a Gaussian with $3 \mathrm{keV}$ standard deviation).

where $k$ is the ratio of the photon energy $E$ and the electron mass energy at rest. Combined with the deposited energy $E^{\prime \prime}$ of (11) for a given deflection angle $\theta$

$$
E^{\prime \prime}=E\left(1-\frac{1}{1+k(1-\cos \theta)}\right)
$$

$\gamma_{0}\left(E, E^{\prime \prime}\right)$ is obtained.

For simplicity, we let $h_{0}\left(E, E^{\prime}\right)$ be a Gaussian distribution with standard deviation $3 \mathrm{keV}$ which is incorrectly modeled by a Gaussian $h$ with standard deviation $4 \mathrm{keV}$. (The Gaussian shape is motivated by previous simulation work showing that charge sharing and pile-up do not lead to a severe left-skewed distribution at reasonable flux [21] and the standard deviation is based on a measured value of $2.2 \mathrm{keV}$ for the pure electronic noise [10] with some margin for the statistical spread around the peak, the pile-up and the charge sharing.) In the below illustration, it is assumed that $\gamma_{0}$ is correctly estimated by $\gamma$.

Two response functions $R_{0}\left(E, E^{\prime}\right)$ and $R\left(E, E^{\prime}\right)$ are depicted in Fig. 4. (That the response function drops for small detected energies around $0 \mathrm{keV}$ is an artefact of the assumed Gaussian form of $h_{0}$ and $h$. This does not matter for the model as the lowest threshold $T_{1}$ is set to $5 \mathrm{keV}$ for rejection of electronic noise.)

\section{METHOD}

Recall that the goal is to find how large misspecifications of the forward model that can be tolerated before the bias component of the mean square error of (4) is larger than the variance part. This appears straight forward: the CRLB of the variance of the $A_{1}$-estimate is given by [7]

$$
\sigma_{\hat{A}_{1}}^{2} \geq \mathcal{F}_{11}^{-1}
$$

where

$$
\mathcal{F}_{j k}=\sum_{i=1}^{N} \frac{1}{\lambda_{i}} \frac{\partial \lambda_{i}}{\partial A_{j}} \frac{\partial \lambda_{i}}{\partial A_{k}}
$$


and the bias is obtained by solution of the maximum likelihood problem given the observed bin counts $\left\{m_{i}\right\}$

$$
\begin{aligned}
A_{1}^{*}, A_{2}^{*} & =\arg \max _{A_{1}, A_{2}} P\left(\left\{m_{i}\right\} ; A_{1}, A_{2}\right) \\
& =\arg \min _{A_{1}, A_{2}} \prod_{i=1}^{N}\left(\lambda_{i}-m_{i} \log \lambda_{i}\right)
\end{aligned}
$$

where $\lambda_{i}=\lambda_{i}\left(A_{1}, A_{2}\right)$ according to (3) [7]. For the bias calculation the expected value of counts in each bin was used, i.e., we apply the approximation $\mathbb{E} \hat{A}\left(\left\{m_{i}\right\}\right) \approx \hat{A}\left(\left\{\lambda_{i}\right\}\right)$.

The results will unfortunately depend on dose and on the size of the region of interest (ROI) that is examined; for larger dose or when averaged over a larger area, the random variance component of (4) will decrease whereas the contribution from the bias will not (at least in the case of a homogenous cylindrical object where the central volume is considered). Thus we have to select typical X-ray fluence for which the comparison is carried out, and also determine how the size of the region of interest affects the result. First however, we show how variance and bias in the projection domain is translated to the reconstructed image domain.

\section{A. Relationship Between $\operatorname{var}(A)$ and $\operatorname{var}(a)$}

Hanson [23] has shown how the single-pixel variance in a reconstructed image depends on the variance of a projection measurement

$$
\sigma_{\hat{a}_{1}}^{2}=\frac{\sigma_{\hat{A}_{1}}^{2}(\lambda)}{N_{\theta} a^{2}} k^{2} .
$$

$a=1 \mathrm{~mm}$ is the size of the detector elements (and distance between samples) and $N_{\theta}$ the number of projection angles. $k$ is a unitless factor depending on filter kernel for the filtered backprojection and determined to $k=0.62$ for MATLAB's iradon with cropped Ram-Lak ramp filter [24]. (The Appendix of [24] contains a general method for determining $k$ of (15).)

\section{B. Relationship Between $\operatorname{bias}(A)$ and $\operatorname{bias}(a)$}

One would easily be lead to believe that the bias in the middle of the reconstructed image of a homogenous cylinder with diameter $L$ is given by bias $(a)=\operatorname{bias}(A) / L$ since by definition

$$
A\left(x^{\prime}, \theta\right)=\int_{l} a(\mathbf{r}) \mathrm{d} s=a L
$$

for a central ray $l$. When estimates are biased, as in the case of a misspecified forward equation, one cannot however be sure that $\hat{A}\left(x^{\prime}, \theta\right)=\hat{a} \int_{l} \mathrm{~d} s$ for all possible path lengths $\left\{\int_{l} \mathrm{~d} s\right\}$ in the sinogram/projection domain. If the relative bias does depend on the path length it is not at all clear how a bias in the projection domain ( $A$-space) translate to the image domain ( $a$-space). The basic problem is similar to a characteristic of the Fourier transform: a change at one point in the Fourier domain of a function alters the spatial representation of the function at all points. The same holds for the filtered back projection due to the filtering step and thus, if relative biases differ across projections for instance due to path length, this will propagate unpredictably to the image domain bias.

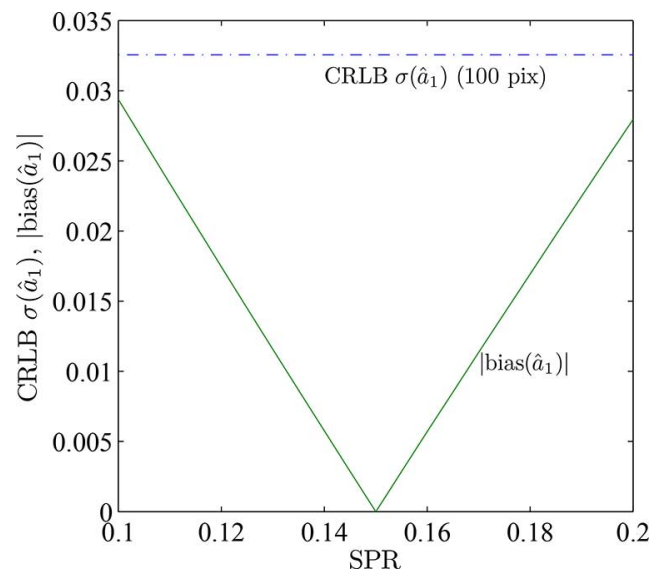

Fig. 5. Effect of errors in the assumed amount of scatter.

A sufficient condition for $\operatorname{bias}(a)=\operatorname{bias}(A) / L$ to hold in the central region of reconstructed image of a homogenous cylinder with diameter $L$ is

$$
\hat{A}=c a \int_{l} \mathrm{~d} s
$$

with constant $c$ for different path lengths $\int_{l} \mathrm{~d} s$. If (17) holds, we have $\operatorname{bias}(A)=\hat{A}-A=(c-1) a \int_{l} \mathrm{~d} s$ since $A=a \int_{l} \mathrm{~d} s$ by definition. In the reconstructed domain, we have $\operatorname{bias}(a)=$ $\hat{a}-a$ where $\hat{a}$ is obtained via the inverse radon transform of $\hat{A}$ of (17). Due to the linearity of the transform, $\hat{a}=c a$ and $\operatorname{bias}(a)=(c-1) a$. Thus, it follows that $\operatorname{bias}(A)=L \operatorname{bias}(a)$.

Equation (17) unfortunately does not hold for all path lengths $\int_{l} \mathrm{~d} s$ and different misspecifications. However, in the Appendix (Fig. 12) we show, via simulation, that $\hat{A} \approx c a \int_{l} \mathrm{~d} s$ for $\int_{l} \mathrm{~d} s \in[0.85,1] L$ for a range of misspecified forward parameters. Since the inverse Fourier transform of the ramp filter (used to convolve the detector response with before back projection) is quite narrow and well centralized, detector readings far away from the center have very little effect on the values in the middle of a reconstructed image. In the present investigation we therefore consider it sufficient to examine the effect of path lengths corresponding to small off center shifts as captured by $\int_{l} \mathrm{~d} s \in[0.85,1] L$. Under these circumstances (17) holds approximately and thus $\operatorname{bias}(a) \approx \operatorname{bias}(A) / L$.

\section{Decrease of Variance as a Function of ROI Area}

The correlation structure in the reconstructed image makes the variance in an ROI consisting of $M$ pixels decaying faster than $M^{-1}$. In the limit of a continuous image, with perfect bandlimited interpolation of the projection data before backprojection, the variance depends on $M$ as $M^{-3 / 2}$ [25]. In previous work [24], we have shown (with a discrete image and typical interpolation) that the variance decreases as $M^{-1.33}$.

For an ROI consisting of $M$ pixels, the standard deviation will thus have decrease by a factor of $M^{-0.66}$. For a $10 \times 10$ pixel $(1 \mathrm{~cm} \times 1 \mathrm{~cm})$ large ROI this evaluates to $100^{-0.66}=$ 0.0479 . Since quantitative CT will most likely be carried out over ROIs much larger than a single pixel, we chose to compare the bias with the variance over a 100 pixel large ROI. 


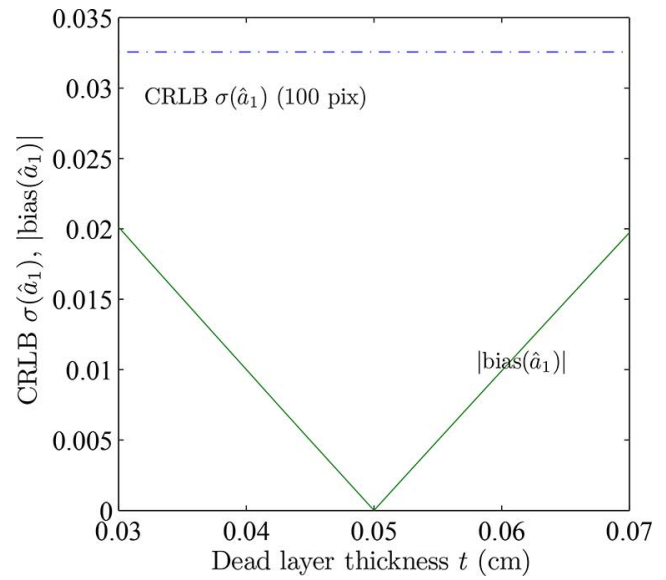

Fig. 6. Effect of errors in the assumed dead layer thickness.

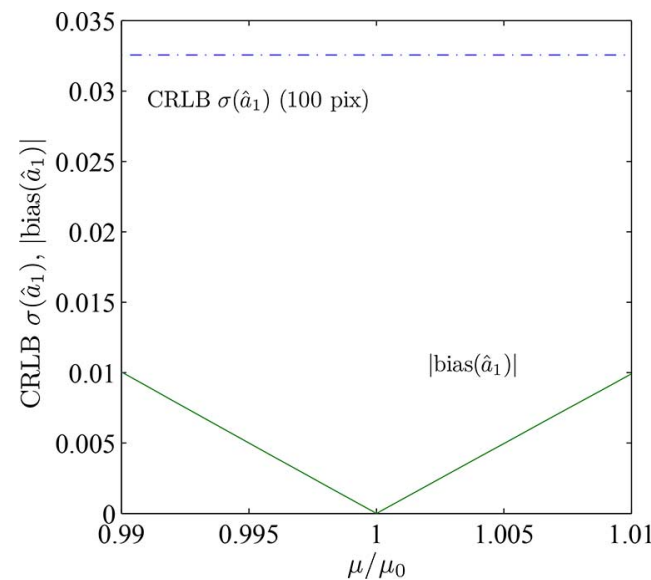

Fig. 7. Effect of errors in the assumed linear attenuation coefficients.

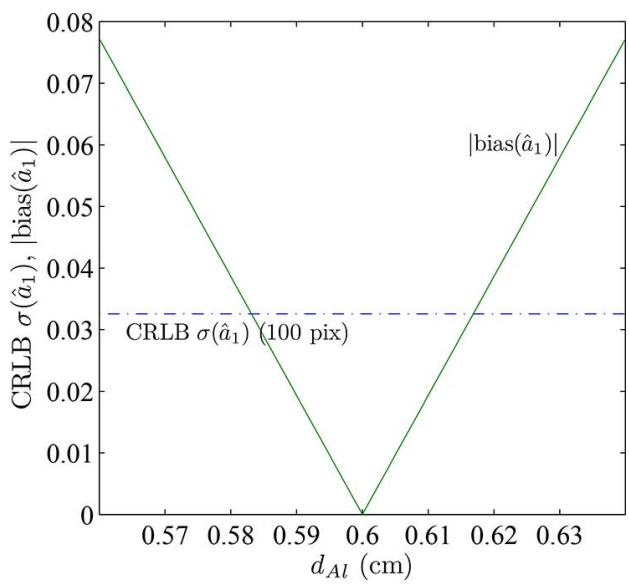

Fig. 8. Effect of errors in the assumed X-ray spectrum stemming from different filtration than assumed.

\section{Assumed Forward Model Parameters}

The homogenous cylindrical object with $L=20 \mathrm{~cm}$ diameter consisting of $50 \%$ soft tissue and $50 \%$ adipose tissue (ICRU 44 [22]). The bases $f_{1}(E)$ and $f_{2}(E)$ of (1) are taken as the linear attenuation coefficients for the same materials. The unattenuated X-ray fluence, $N_{0}$, must be selected as the total number of X-rays per reconstructed pixel area for an entire

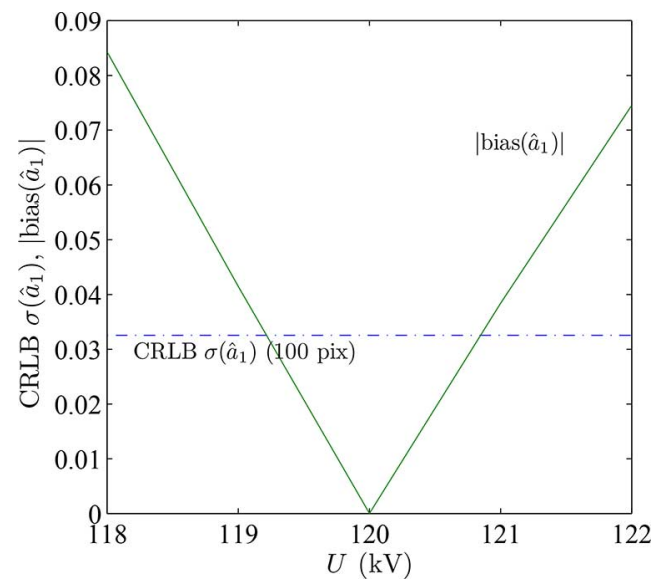

Fig. 9. Effect of errors in the assumed X-ray spectrum stemming from a different acceleration voltage than assumed.

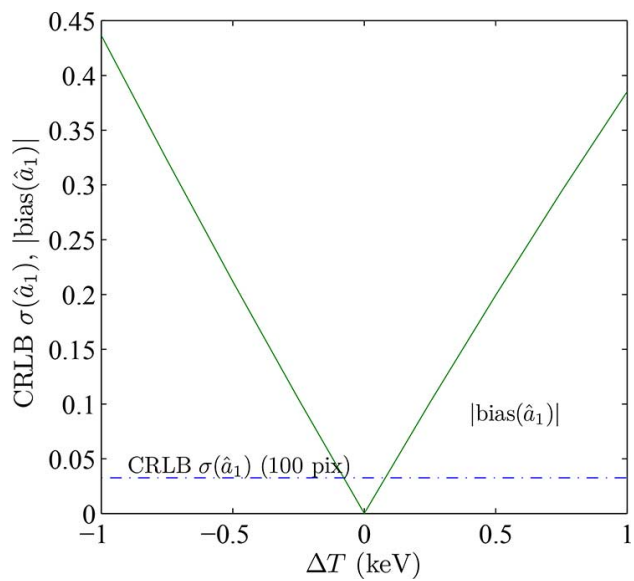

Fig. 10. Effect of uncertainty in thresholds.

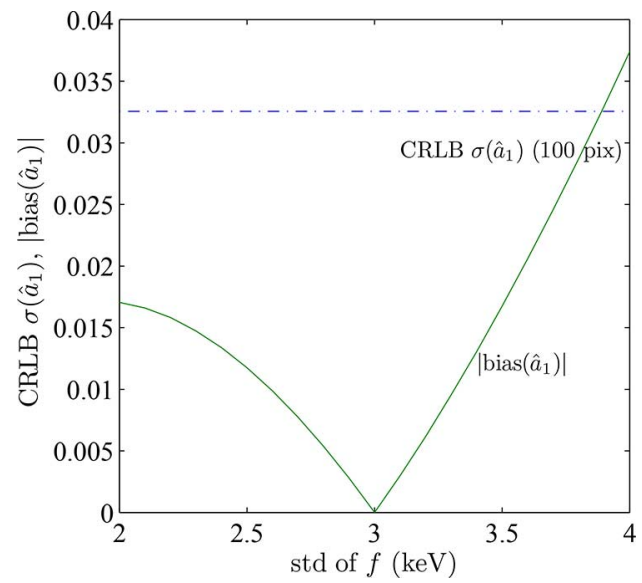

Fig. 11. Effect erroneous response function.

gantry revolution. (Note that the lower bound of the variance in the reconstructed image is independent of the number of angles and depends only on $N_{0}$ since $\lambda=\lambda\left(N_{0} / N_{\theta}\right)$ in (12) and $\sigma_{\hat{A}_{1}}^{2}\left(\lambda\left(N_{0} / N_{\theta}\right)\right) / N_{\theta}=\sigma_{\hat{A}_{1}}^{2}\left(\lambda\left(N_{0}\right)\right)$.) Assuming a typical current of $360 \mathrm{~mA}$ the X-ray tube model of Cranely et al. [16] with a $120 \mathrm{kVp}$ tungsten anode spectrum, $6 \mathrm{~mm} \mathrm{Al} \mathrm{filtration,} 7^{\circ}$ anode angle and $1000 \mathrm{~mm}$ source-to-isocenter distance gives $8.1 \cdot 10^{8}$ photons $\mathrm{mm}^{-2} \mathrm{~s}^{-1}$ on the detector. With 3 revolutions 

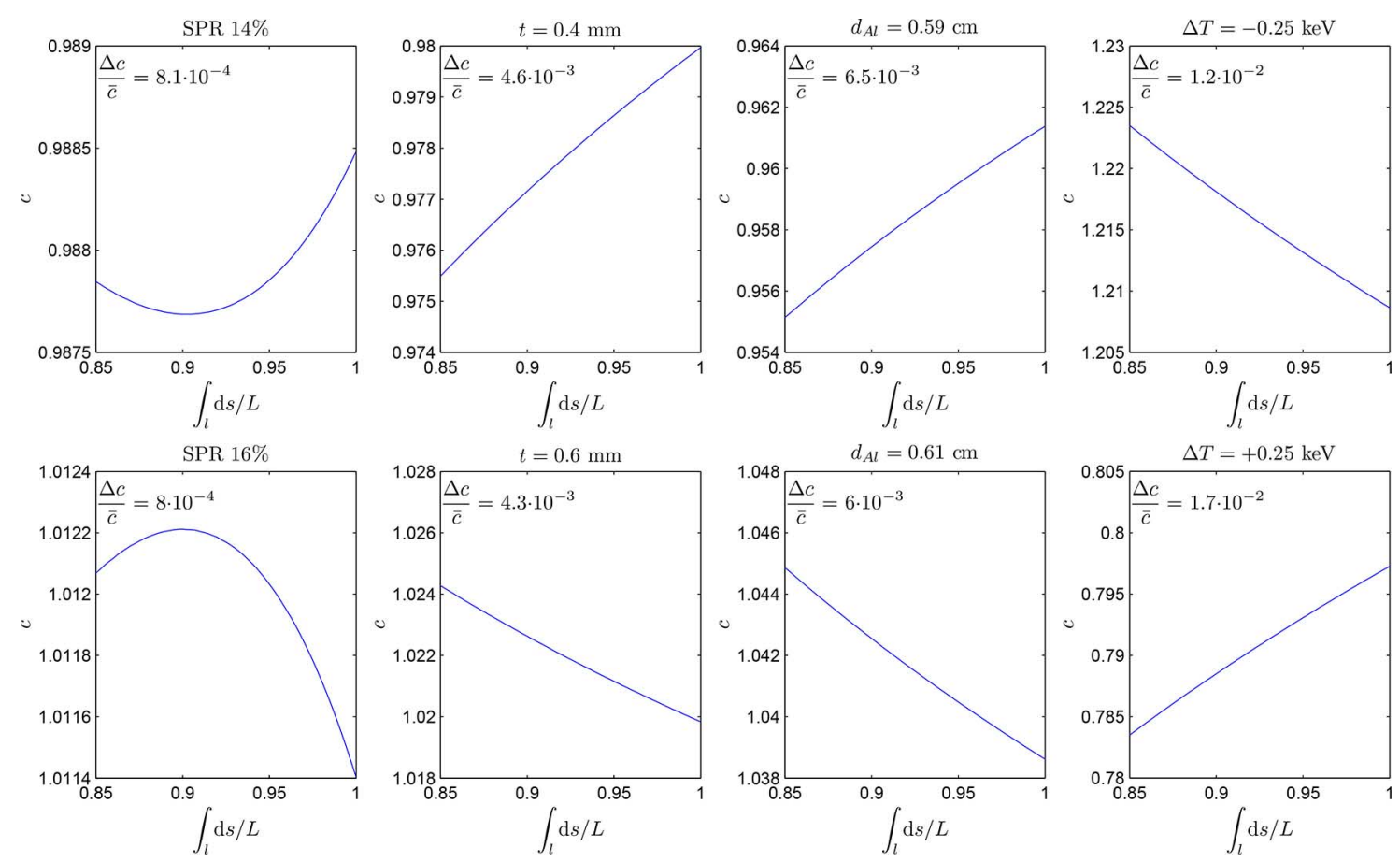

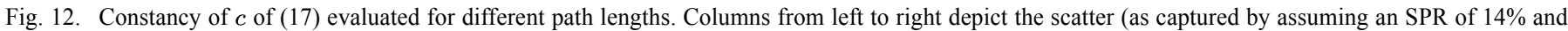

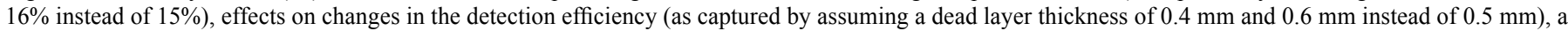
change in input spectrum from varying the aluminum filtration and, last column, a change in threshold values. $\Delta c=\max (c)-\min (c)$.

per second as a typical rotation speed $N_{0}=2.7 \cdot 10^{8}$ are directed towards a central pixel area of $1 \mathrm{~mm} \times 1 \mathrm{~mm}$ (which is the area henceforth assumed).

For the default parameter values, the Cramér-Rao lower bound of the variance of the $A_{1}$-estimate is given by (12) and (13) in conjunction with (3). Combined with (15) this yields $\sigma\left(\hat{a}_{1}\right)=0.68$ (unitless) (which should be related to $\mathbb{E}\left(\hat{a}_{1}\right)=0.5$ (also unitless)). For an ROI with $M=100, \sigma\left(\hat{a}_{1}\right)$ is 0.033 . This is the value the bias should be compared to.

\section{REsults AND DisCUSSION}

\section{A. Effect of Uncertainty in Scatter Counts $\varepsilon_{i}\left(x^{\prime}, \theta\right)$}

In Fig. 5, the bias resulting from misspecification of the scatter counts is illustrated. When large regions are considered the misspecifications can be rather large $(0.2$ instead of 0.15 is a $33 \%$ error) before the bias has a significant effect on the MSE.

\section{B. Effect of Uncertainty of the Detection Efficiency $D(E)$}

In Figs. 6 and 7, the result of misspecification of the detection efficiency by erroneous dead layer assumption and faulty linear attenuation coefficients. Clearly, the quantum noise will dominate the error for realistic ROIs and errors of the forward model.

\section{Effect of Uncertainty of the X-Ray Spectrum $\Phi(E)$}

That basis decomposition is very sensitive to assumptions regarding X-ray tube acceleration voltage is known [6]. Our result, illustrated in Figs. 8 and 9, confirms this. While the thickness of any aluminum filter can certainly be determined with a very high accuracy, the actual X-ray tube voltages can easily differ by a few $\mathrm{kV}$ from stipulated values. This in itself is not a problem, since the actual X-ray spectra for each tube setting can be accurately measured. Instead it is the reproducibility of the voltage that is critical, and typically modern $\mathrm{X}$-ray tubes reproduce the peak voltage with an uncertainty of only $0.01 \%$. Thus Fig. 9 indicates that the bias introduced this way will be dominated by the statistical error in the quantitative determination of the basis function coefficients $a_{1}$ and $a_{2}$.

\section{Effect of Uncertainty of the Reference Thresholds $\left\{T_{i}\right\}$}

Fig. 10 indicates that misspecified thresholds are very detrimental to quantitative CT. For parallel shifts in the thresholds of $0.1 \mathrm{keV}$ or more (as compared to assumed values in the forward model), the bias introduced will dominate the MSE. This indicates that thresholds uncertainties of much more than $0.15 \mathrm{keV}$ cannot be tolerated for quantitative $\mathrm{CT}$ if threshold uncertainties all line up in the same direction (otherwise the effect will be less serious) and if it is deemed essential to perform the decomposition at the quantum limit (where statistical noise makes up the lion's share of the mean square error). At the very least, the level is indicative of what good calibration methods should strive for.

\section{E. Effect of Uncertainty of the Response Function $R\left(E, E^{\prime}\right)$}

In obtaining $R\left(E, E^{\prime}\right)$ of Fig. $4, h\left(E^{\prime}, E^{\prime \prime}\right)$ was assumed to be Gaussian with a standard deviation of $4 \mathrm{keV}$ instead of $3 \mathrm{keV}$. The resulting bias for different choices of the Gaussian standard deviation is shown in Fig. 11. Clearly, for reasonable deviations of $h$ from $h_{0}$, the statistical limitation (variance) outweighs the bias. 


\section{CONCLUSION}

We have presented a method to determine allowable misspecification in based photon counting multibin system under the assumption that one wants to perform close to quantum limited quantitative CT. With quantum limited we mean that the statistical noise should dominate over the bias introduced by the misspecifications. We have also quantified the allowable misspecifications for a particular silicon strip detector system. The results show that the bin edges are the most critical parameters of the forward model and that they need to be determined with an accuracy of $0.15 \mathrm{keV}$ given the assumptions of this particular investigation (silicon detector and thresholds that all shift in parallel). Devising calibration methods, preferably not relying on synchrotron radiation, whereby the thresholds of individual channels can be determined accurately is therefore very important to be able to reap the expected benefits of spectral CT.

\section{APPENDIX}

In this section we show that $c$ of (17) is close to constant for some different forward model misspecifications. For values of the path length $\int_{l} \mathrm{~d} s$ ranging from $0.85 L$ to $L, \hat{A}_{1}$ is estimated by (14) [by insertion of the erroneous forward parameter in (3)]. $c$ is then determined by $c=\hat{A}_{1} / \int_{l} a_{1} \mathrm{~d} s$ and plotted against path length expressed as a ratio to the diameter $L$ in Fig. 12 . Relative changes of $c$ seem to be confined to around or less than $1 \%$ over the interval, for which reason we can model it as approximately constant.

\section{REFERENCES}

[1] R. E. Alvarez and A. Macovski, "Energy-selective reconstruction in X-ray computerized tomography," Phys. Med. Biol., vol. 21, no. 5, pp. 733-44, 1976.

[2] L. A. Lehmann, R. E. Alvarez, A. Macovski, W. R. Brody, N. J. Pelc, S. J. Riederer, and A. L. Hall, "Generalized image combinations in dual kVp digital radiography," Med. Phys., vol. 8, no. 5, pp. 659-667, 1981.

[3] J. E. Tkaczyk, D. Langan, X. Wu, D. Xu, T. Benson, J. D. Pack, A. Schmitz, A. Hara, W. Palicek, P. Licato, and J. Leverentz, "Quantization of liver tissue in dual $\mathrm{kVp}$ computed tomography using linear discriminant analysis," in Proc. SPIE, 2009, vol. 7258, p. 72580G.

[4] P. C. Johns and M. L. Yaffe, "Theoretical optimization of dual-energy X-ray imaging with application to mammography," Med. Phys., vol. 12, no. 3, pp. 289-296, 1985 .

[5] R. E. Alvarez, "Estimator for photon counting energy selective X-ray imaging with multibin pulse height analysis," Med. Phys., vol. 38, no. 5, pp. 2324-2334, 2011.

[6] D. J. Walter, E. J. Tkaczyk, and X. Wu, "Accuracy and precision of dual energy CT imaging for the quantification of tissue fat content," in Proc. SPIE, 2006, vol. 6142, p. $61421 \mathrm{G}$.
[7] E. Roessl and C. Herrmann, "Cramér-Rao lower bound of basis image noise in multiple-energy X-ray imaging," Phys. Med. Biol., vol. 54, no. 5, pp. 1307-1318, 2009.

[8] E. Roessl and R. Proksa, "K-edge imaging in X-ray computed tomography using multi-bin photon counting detectors," Phys. Med. Biol., vol. 52, no. 15, pp. 4679-4696, 2007.

[9] H. Bornefalk and M. Danielsson, "Photon counting spectral computed tomography using silicon strip detectors: A feasibility study," Phys. Med. Biol., vol. 55, no. 7, pp. 1999-2022, 2010.

[10] H. Bornefalk, M. Persson, C. Xu, S. Karlsson, C. Svensson, and M. Danielsson, "Effect of temperature variation on the energy response of a photon counting silicon CT detector," IEEE Trans. Nucl. Sci., vol. 60, no. 2, pp. 1442-1449, Apr. 2013.

[11] C. Xu, M. Danielsson, S. Karlsson, C. Svensson, and H. Bornefalk, "Preliminary evaluation of a silicon strip detector for photon-counting spectral CT," Nucl. Instrum. Methods Phys. Res., Sect. A, vol. 677, pp. 45-51, 2012.

[12] C. Xu, M. Persson, H. Chen, S. Karlsson, M. Danielsson, C. Svensson, and H. Bornefalk, "Evaluation of a second-generation ultra-fast energy-resolved ASIC for photon-counting spectral CT," IEEE Trans. Nucl. Sci., vol. 60, no. 1, pp. 437-445, Feb. 2013.

[13] H. Bornefalk, M. Persson, and M. Danielsson, "Necessary forward model specification accuracy for basis material decomposition in spectral CT," in Proc. SPIE, 2014, vol. 9033, p. 90332I.

[14] J. H. Hubbell, "Photon mass attenuation and energy-absorption coefficients," Int. J. Appl. Radiat. Isotopes, vol. 33, no. 11, pp. 1269-1290, 1982.

[15] M. J. Berger, J. H. Hubbell, S. M. Seltzer, J. Chang, J. S. Coursey, R. Sukumar, and D. S. Zucker, 2005 XCOM: Photon Cross Section Database ver. 1.2. Gaithersburg, MD, Nat. Inst. Standards Technol. [Online]. Available: http://physics.nist.gov/xcom

[16] K. Cranley, B. J. Gilmore, G. W. A. Fogarty, and L. Desponds, Catalogue of diagnostic X-ray spectra and other data IPEM Rep. 78 (York: IPEM), 1997, 1997.

[17] M. Gustavsson, F. U. Amin, A. Björklid, A. Ehliar, C. Xu, and C. Svensson, "A high-rate energy-resolving photon-counting ASIC for spectral computed tomography," IEEE Trans. Nucl. Sci., vol. 59, no. 1, pp. 30-39, Feb. 2012

[18] M. Persson, B. Meyer, H. Bornefalk, and M. Danielsson, "Quantification of ring artifact visibility in CT," in Proc. SPIE, 2012, vol. 8313, p. 83132J.

[19] M. Persson and H. Bornefalk, "A framework for evaluating threshold variation compensation methods in photon counting spectral CT," IEEE Trans. Med. Imag., vol. 31, no. 10, pp. 1861-1874, Oct. 2012.

[20] A. Owens, G. W. Fraser, and K. J. McCarthy, "On the experimental determination of the Fano factor in Si at soft X-ray wavelengths," Nucl. Instrum. Methods Phys. Res., Sect. A, vol. 491, pp. 437-443, 2002.

[21] H. Bornefalk, C. Xu, C. Svensson, and M. Danielsson, "Design considerations to overcome cross talk in a photon counting silicon strip detector for computed tomography," Nucl. Instrum. Methods Phys. Res., Sect. A, vol. 621, pp. 371-378, 2010.

[22] ICRU Rep. 44 Int. Commiss. Radiat. Units Measur., Bethesda, MD, 1989.

[23] K. M. Hanson, "Detectability in computed tomographic images," Med. Phys., vol. 6, no. 5, pp. 441-451, 1979.

[24] H. Bornefalk and M. Persson, "Theoretical comparison of the iodine quantification accuracy of two spectral CT technologies," IEEE Trans. Med. Imag., vol. 33, no. 2, pp. 556-565, Feb. 2014.

[25] S. J. Riederer, N. J. Pelc, and D. A. Chesler, "The noise power spectrum in computed X-ray tomography," Phys. Med. Biol., vol. 23, no. 3, pp. 446-454, 1978. 